\title{
Thermic Modeling of Unheated Tunnel - Greenhouse
}

\author{
F. Sahbani ${ }^{1}$, A. Chbeb $^{2}$, E. Ferjani ${ }^{3}$ \\ ${ }^{1,3}$ Bizerte Faculty, Department of vegetable biology, University of Carthage, Tunisia \\ ${ }^{2}$ Engineering School of Bizerte (INIB), University of Carthage ,Tunisia
}

\begin{abstract}
Tunisia, we encounter major problems of heating, air-conditioning and diseases in particular the mildew. As a remedy to these problems, a physical model, based on analysis of the assessment of energy, is developed to estimate the requirement in heating for agricultural greenhouse tunnel. Another model based on the climatic data makes it possible to estimate the relation between the temperature and the relative humidity inside the greenhouse with an aim of fighting against the disease of the mildew thanks to ventilation. In other term, a compromise between the temperature and the relative humidity inside the greenhouse must be established.
\end{abstract}

Keywords: Greenhouse, Energy, Heating, Relative Humidity.

\section{Introduction}

of the developed models. The relation of the nonlinear equations thus developed was made possible only by the linearization of the equations translating the energy assessments.

The linearization of these equations remains only one good solution to solve these mathematical equations. The greenhouse as a system is complex as the linearization yet unable to solve it. The model "limps black" seems satisfactory by introducing a mathematical formulation of its behavior entries-left Currently in Tunisia, the agricultural greenhouses have a paramount importance so far as they contribute to the creation of a microclimate, in terms of hygrometrical moisture and temperature of vegetable cover and interior air, favorable to the needs of the tomato plant. These greenhouses are generally designed ways to control mainly the temperature, the relative humidity of the air inside the greenhouse, solar radiations and the content carbon dioxide. Indeed, the share of the solar radiations transmitted inside the greenhouse influences perspiration, photosynthesis, the speed of growth and the quality of the tomato product [1].

Furthermore, the share of research, one does not find a relation between the needs for the tomato plant in energy, hygroscopy and the diseases which are due to the lack or excess of these needs. The reader will be able to refer in particular to work of Jolliet[2] (1988), Monteil et al (1991)[3]; Boulard et al (1993)[4]. Because of the lack of absence of the expert agronomists and energy at the same time, the farmers use empirical formulas inherited by dress use.

The calculation of the couple of relative temperature-moisture of the air inside the agricultural greenhouses using the concept of perspiration introduced by Stanghellini (1987)[5], dominated the majority or the entries are the instructions and the disturbances of the system and the exits are the measurements taken by sensors.

\section{A. Application of the Biophysics System of The Greenhouse}

\section{Energy Modeling Of An Agricultural Greenhouse}

In this section, we present the theoretical and physical contributions to the modeling of the greenhouses. Identification of the parameters of the assessments of energy and mass $\left(\mathrm{H}_{2} \mathrm{O}, \mathrm{CO}_{2}\right)$ of the greenhouse. The use of the techniques of identification of the dynamic systems rests on approach of analysis of the heat transfers calling upon the mathematical formalisms according to: vector $\mathrm{X}(\mathrm{t})$ of the variables characterizing the dynamic state of the system is connected to the vector $U(t)$ exogenic sizes and to the vector $\mathrm{Y}(\mathrm{t})$ of the variables of observation of the system.

The transposition of this approach to the case of the agricultural greenhouses encounters certain difficulties, related to the close coupling between transfer of significant heat and latent heat and to the representation of the operation of cover (perspiration and photosynthesis of the plants). In our study, the system greenhouse-vegetation will be analyzed through the variables table I below. The general form of the equations binding these various variables is represented in what follows.

\section{B. Model of Knowledge To The Small-Scale Model}

The choice of a behavior model with a reduced number of parameters can be done on the basis of the following consideration: 
- First of all, an approach of the type "singular disturbances" makes it possible to consider that the air and the vegetation evolve/move of thermal sight as if they were deprived of inertia (this approximation is related to the choice of the step of observation used, namely 1:00).

- The interval of sampling is one o'clock during the day of April $25^{\text {th }}, 2015$ where we have made the taking of the necessary measures thanks to the following materials (equipment):

- A sensor of temperature who presents a precision of $\pm 0.5^{\circ} \mathrm{C}$. It is about LM35DZ.

- A sensor of type HMP35AC used for the taking of the measure of relative humidity.

- An anemometer of precision of $\pm 5 \%$ to detect the wind speed.

- A sensor SKP215 quantum sensor used for the measure of illumination.

- These sensors are placed in the middle of the culture of tomato at the level of the height of the plant.

- Then, the "heavy" evolution of the parts thermically of greenhouse (ground, carrying structures, etc), can be from the dynamic point of view approximated by a dynamic system of first order. It leaves there that the writing of the various assessments can be done by considering the following elements:

- The thermal and virtual mass (heavy ground and elements), characterized by its temperature Tm and its heat-storage capacity $\mathrm{Cm}$;

- Air and vegetation, which is also the seat of evaporative exchanges and $\mathrm{CO}_{2}$, gathered in only one element whose heat capacity is negligible, which element is characterized by its resultant temperature Ti, its steam pressure of water ei. (internal excitation), and its $\mathrm{CO}_{2}$ concentration, $\mathrm{Ci}$. The mathematical model of the system is then obtained by writing the assessments of energy and mass to each one of its nodes.

\section{Assessments Of Energy}

Mass thermal virtual such as data by figure 1.The thermal variation of energy in the system $\mathrm{Cm}$ $(\mathrm{dTm} / \mathrm{dt})\left(\mathrm{wm}^{-2}\right)$, is the resultant:

. solar profit, $\beta \mathrm{Rg}, \beta$ are the absorption coefficient of the external total radiation by the thermal mass;

. exchanges with the air of the greenhouse, $\mathrm{h}(\mathrm{Ti}-\mathrm{Tm})$;

. contributions of heating of ground or substrate, Qsol.

. from where $\mathrm{Cm}(\mathrm{dTm} / \mathrm{dt})=\mathrm{h}(\mathrm{Ti}-\mathrm{Tm})+\mathrm{Qsol}+\mathrm{bRg}$

In the equation relating to the assessment of air, one neglects the heat-storage capacity of the behavior

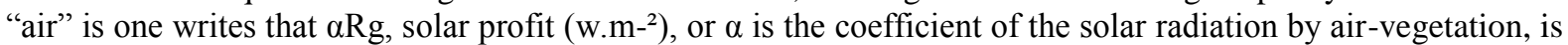
balanced by:

. Energy of heating, Ch;

. Exchanges with the thermal mass, h(Tm-Ti);

. Exchanges through the wall, $\mathrm{K}(\mathrm{Te}-\mathrm{Ti})$;

- $\quad$ Significant heat transfers by ventilation and escapes, Ks (Te-Ti);

- latent heat transfers by ventilation and escapes, $\operatorname{Ke}\left(\mathrm{e}_{0}-\mathrm{ei}\right)$.(internal excitation)). In these the last two terms, $\mathrm{Ks}=\rho \mathrm{CpG} / \mathrm{S}\left(\mathrm{wm}^{-}{ }^{2} \mathrm{k}^{-1}\right.$ and $\mathrm{Kl}=\rho \mathrm{CpG} / \mathrm{YS}$, are coefficients of transfer which characterize the significant and latent losses of heat per infiltration or ventilation, proportional to the volume throughput of air broken down G (BOULARD and al.1996).

From where: $\alpha \mathrm{Rg}+\mathrm{Ch}+\mathrm{h}(\mathrm{Tm}-\mathrm{Ti})+\mathrm{K}(\mathrm{Te}-\mathrm{Ti})+\mathrm{Ke}(\mathrm{eo}-\mathrm{ei}) .=0$

- Concerning the steam assessment, the variation of the latent steam energy of the "compartment air", $\mathrm{Cl}$ $\mathrm{dei} / \mathrm{dt}\left(\mathrm{wm}^{-2}\right)$, is equal to the resultant:

- Latent heat transfers by ventilation and escapes, $\mathrm{Cl}=\rho \mathrm{CpVs} / \Upsilon \mathrm{S}$, being equivalent heat-storage capacity of the steam of the air;

- $\quad$ Latent energies of the water perspired by culture (Ec) and daughter-in-law mized in greenhouse (Br).

Perspiration is given in a way simplified according to the total radiation under greenhouse, Rgi, and of the deficit of saturation of the air of the greenhouse:

Di: $\mathrm{Ec}=$ Argi $+\mathrm{BDi}$

From where: $\mathrm{Cl}(\mathrm{dpi} / \mathrm{dt})=\mathrm{Ec}-\mathrm{Kl}(\mathrm{pi}-\mathrm{pe})+\mathrm{Br}$

By using the assessment $\mathrm{CO}_{2}$ of the air of the greenhouse, one can say that variation of the quantity of $\mathrm{CO}_{2}$ in the greenhouse:

Vs dCi/Ss dt $\left(\mathrm{gCo}^{-} \mathrm{m}^{-} \mathrm{s}^{-1}\right)$ is equal to the resultant:

- $\mathrm{CO}_{2}$ losses and contributions;

- $\quad$ Losses of $\mathrm{CO}_{2}$ by ventilation and escapes, $\mathrm{G}(\mathrm{Ci}-\mathrm{Co}) / \mathrm{S}$;

- Losses due to clear photosynthesis $\mathrm{ScPn} / \mathrm{Ss}$;

- Profits by enrichment, En, from where:

$\mathrm{Vg} / \mathrm{S}(\mathrm{dCi} / \mathrm{dt})=\mathrm{Sc} \mathrm{Pn} / \mathrm{S}-\mathrm{Gv} / \mathrm{S}(\mathrm{Ci}-\mathrm{Co})+\mathrm{En}$ 


\section{Thermal And Climatic Analyses}

After having installed the transmitters of temperature, of relative humidity, speed of the wind, heat flow, light radiation and a stop watch to measure time that the experiment occupied, one took the necessary measures with the establishment of the graphs. In our application, one used the sensor of the type HMP35AC which measures at the same time the temperature and the relative humidity. Other factors are supposed to be constant and negligible by reports/ratios with those which are necessary to the realization of our research. Among these factors, let us quote the pressure. These measurements seem to us necessary and sufficient to the realization of the graphs which follow.

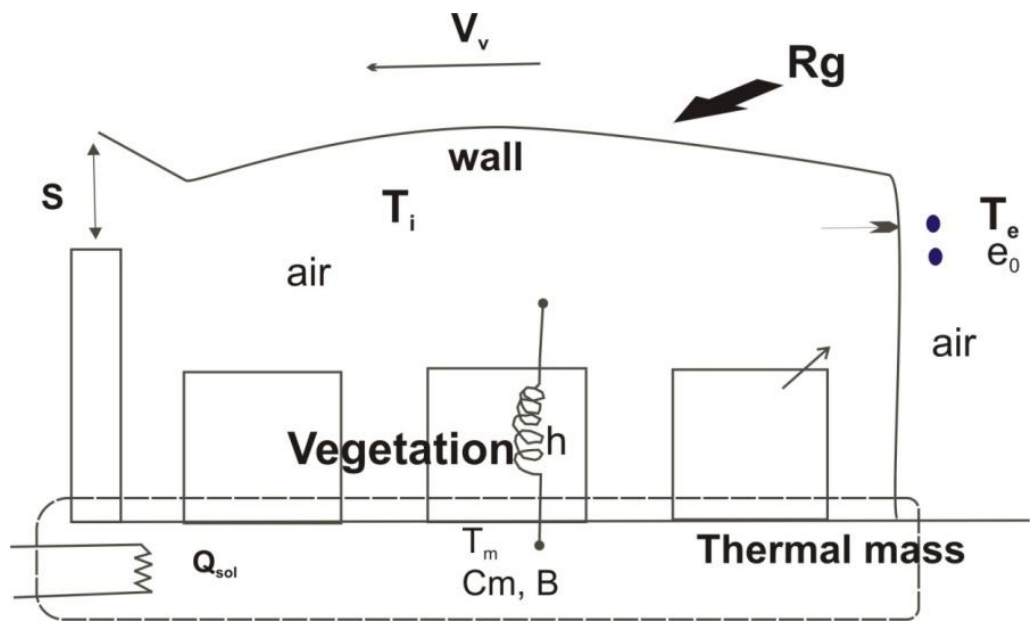

Fig. 1. Lay-out of the balance-sheet of energy of the thermal mass (reference)

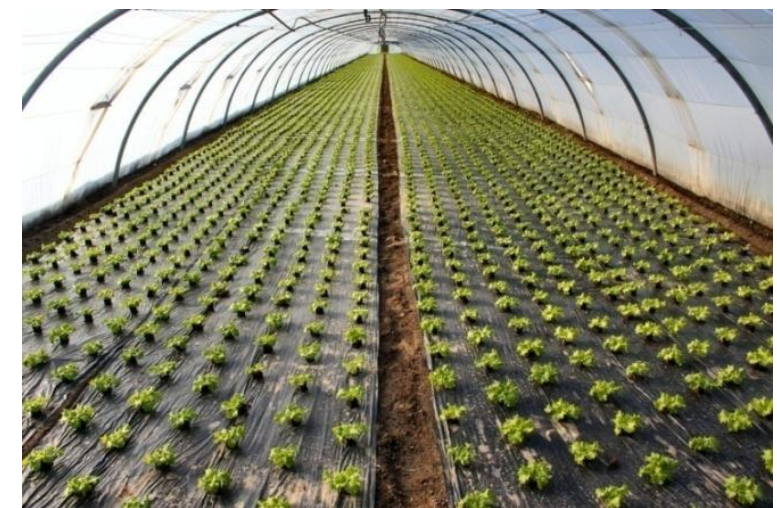

Fig. 2. Agricultural tunnel greenhouse (https://fr.fotolia.com/id/10107450)

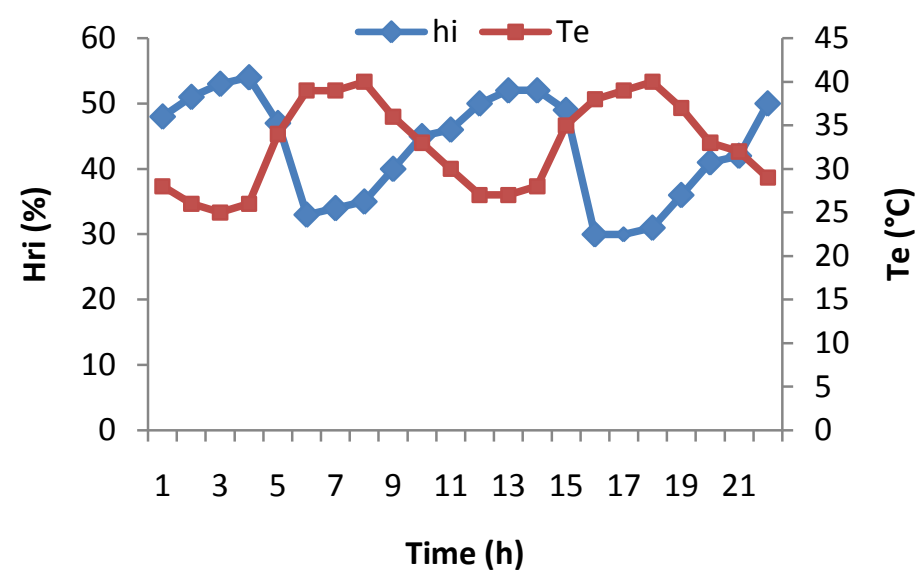

Fig. 3. Variation of the relative humidity interns (Hri) and the external temperature (Te) according to time. Measurements were done during a representative day of April $25^{\text {th }}, 2015$. 


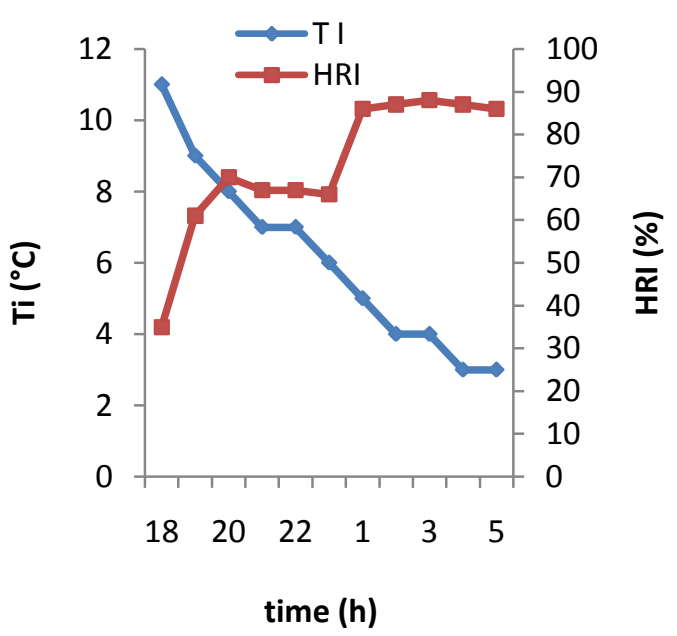

Fig. 4. Variation of the relative humidity interns (Hri) and the internal temperature (Ti) according to time.

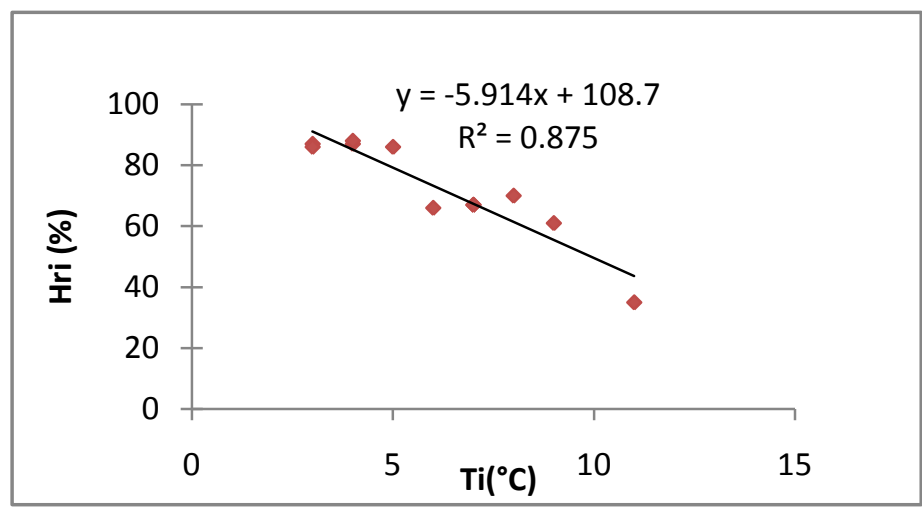

Fig. 5.Variation of the relative humidity interns (Hri) according to the internal temperature (Ti).

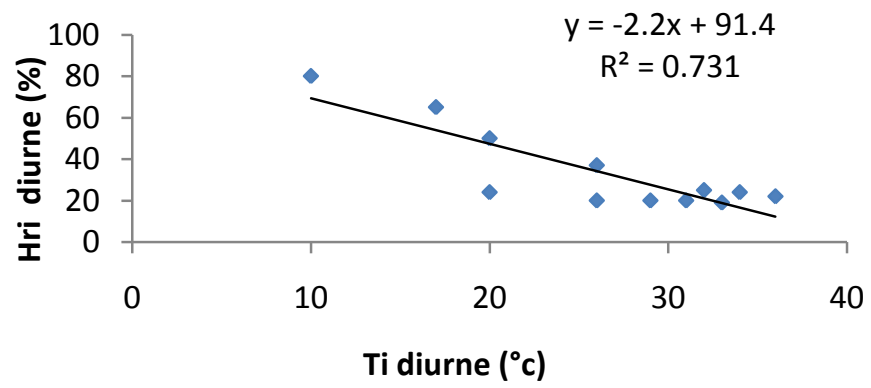

Fig. 6. Relative variation of the humidity interns diurnal (Hri) according to the diurnal internal temperature (Ti)

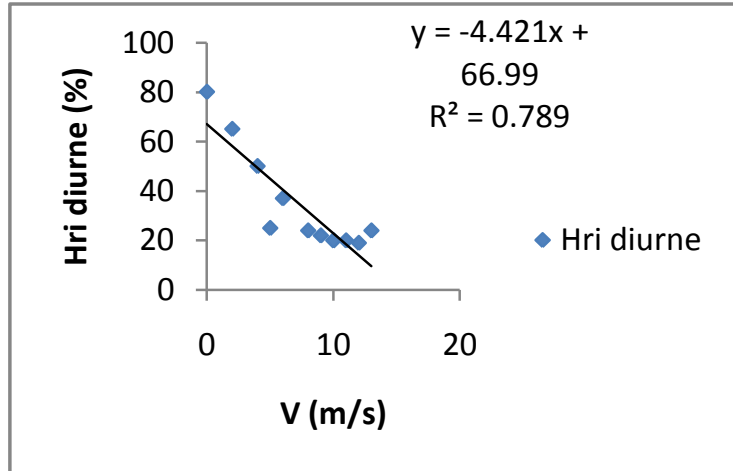

Fig. 7. Variation of the relative humidity interns diurnal (Hri) according to the speed of the wind. 


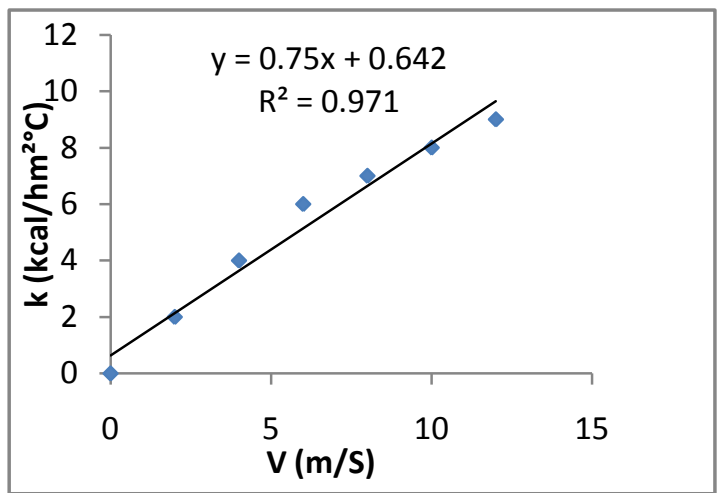

FIG. 8 Variation of the total coefficient of thermal loss (K) according to the speed of the wind.

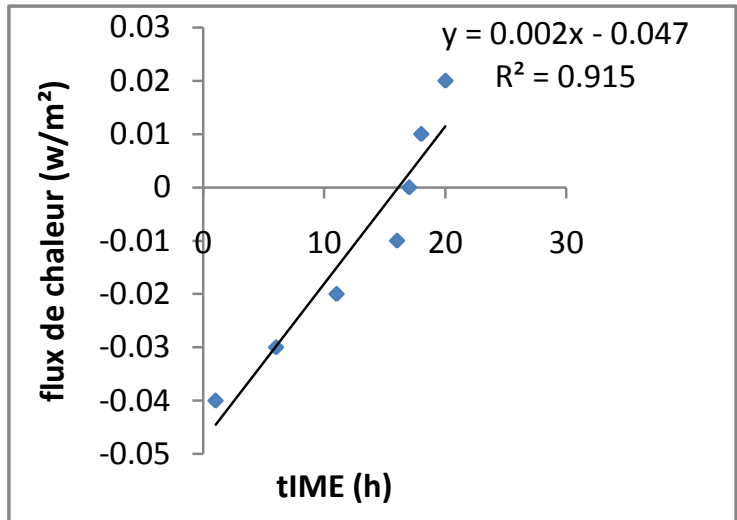

FIG. 9 Variation of the heat flow according to time

\section{Interpretations of experimental results}

After steps, figure 3 shows that the internal relative humidity is inversely proportional to the external temperature of the greenhouse and at the same time dependent on the time (day and night).It reaches a maximum when the external temperature is minimal. The same situation is found in figure 4 . The coefficient of determination $\mathrm{R}^{2}$ reflects the dispersion of the cloud of points around the regression line. The coefficient of determination is used to identify the validity of this equation. It is a measure of the variance of the internal relative humidity «is explained » by changes in the variable on the abscissa. Accordingly, it is possible to predict $73,1 \%$ of the variability based on the relationship with the values of internal temperature of the greenhouse.

On figure 7, the relative humidity interns diurnal is attenuated by the ventilation (speed of the wind) produced by opening greenhouse. Consequently, one can predict that $84.5 \%$ of the variability of the values of moisture while being based on the relationship to the values of the natural speed. The result of these regressions (figure 8 ) shows us a positive correlation $\left(\mathrm{R}^{2}=0.971\right)$. This result enables us to confirm that the total coefficient of thermal loss is closely related to the speed of the wind entering to the greenhouse to air the latter.

\section{Conclusion}

The work presented in this article made it possible to characterize the evolution of the total coefficient of heat transfer through the wall of the greenhouse. The samples of curves materialize the evolution of the coefficient (K) according to the speed of the wind. They assure the absence of linearity of this coefficient $(\mathrm{K})$. This last evolving/moving according to the speed of the wind noted $\mathrm{K}=\mathrm{a}+\mathrm{bV}$ used a long time in our calculation is a linear line. It over-estimates the values of this coefficient $(\mathrm{K})$. This over-estimation involves a swelling of the heat flows through the greenhouse. The relation binding the relative humidity of the air of the greenhouse to the internal temperature is $\mathrm{Y}=\mathrm{aX}+\mathrm{b}$. This last relation, as a farmer, seems necessary to me in order to intervene me in the event of disease of mildew at the plants. To fight against this disease, a compromise, between the internal temperature of the greenhouse and the relative humidity, must be established thanks to ventilation. This relation is often absent in all researches. The absence of mathematical linear relation, binding the temperature, moisture interns and ventilation carries out us to start a research project for the order of these 3 variables by logic fuzzy and by helping in of the ordering of the system "limps black" or left entries. The requirement in heating is primarily night. 
There is a narrow relation between the energy, the climate and the sanitary state of the plant. Indeed, it was very hot during the April in Tunisia in the daytime and it was very cold at night from 2am with condensation of the droplets of water. Further to this climate, the plant of tomato begins by (with) drying then dying. Usually, if this disease is really the one of the "mildew" we can fight against by a chemical treatment (processing) in case when the aeration is not effective. This time this, in spite of the chemical preventive and curative treatment (processing), the disease continues to attack (affect) the culture of tomato. This inappropriate disease appeared this year in china in the culture of tomatoes and peppers. The Chinese researchers asserted that this disease is the resultant of an abrupt change of the climate.

\section{APPENDICES}

Table I: Characteristics of the variables intervening in the assessments of energy and mass of a greenhouse

\begin{tabular}{|c|c|c|}
\hline Entries $U(t)$ & Left $Y(t)$ & State $X(t)$ \\
\hline $\begin{array}{l}\mathrm{Rg}: \text { total radiation } \\
\mathrm{Te}: \text { outside temperature } \\
\text { Qsol : flow of the ground heating } \\
\mathrm{Ch}: \text { flow of air heating } \\
\mathrm{Vv}: \text { speed of the wind } \\
\mathrm{S}: \text { open surface of opening } \\
\mathrm{Br}: \text { energy absorbed by brumisation } \\
\mathrm{e}_{\mathrm{o}}: \text { steam pressure of water of the surrounding air } \\
\mathrm{Tr}: \text { flow of perspiration of the culture } \\
\mathrm{Pn}: \text { flow Net of } \mathrm{CO}_{2} \text { absorbed or rejected by the } \\
\text { culture } \\
\mathrm{Co}: \mathrm{CO}_{2} \text { concentration external in the greenhouse } \\
\text { En : } \mathrm{CO}_{2} \text { flow of enrichment }\end{array}$ & $\begin{array}{l}\text { Ti : temperature of } \\
\text { the interior air } \\
\text { ei : steam pressure } \\
\text { of water of the } \\
\text { interior air } \\
\mathrm{Ci} \text { : } \\
\text { concentration } \mathrm{CO}_{2} \\
\text { under } \\
\text { greenhouse }\end{array}$ & $\begin{array}{l}\text { Tm: temperature of the } \\
\text { thermal mass } \\
\text { Ti: temperature of the } \\
\text { interior air } \\
\text { ei: steam pressure of the } \\
\text { interior air } \\
\mathrm{Ci}: \mathrm{CO}_{2} \text { concentration } \\
\text { under the greenhouse }\end{array}$ \\
\hline
\end{tabular}

\section{References}

[1]. S. Kania S, Giacomelli G. 2001. Solar Radiation Availability For plant Growth in Arizona controlled Environment Agriculture System ; college of Agriculture and Life science, the University of Arizona, CEAC, paper=p-125933-08-014, CEAC.

[2]. Jolliet O. 1988. Modélisation du comportement thermique d'une serre horticole, Thèse de docteur Ingénieur, Ecole polytechnique Fédérale de Lausanne.

[3]. Monteil C, Issachou G, Amoureux M. 1991. Modèle Energétique de la serre Agricole, Journal de physique3, vol.1, No3, pp.429454.

[4]. Boulard T, Baille A. 1993. A simple Greenhouse climate Model Incorporation Effects of Aération and Evaporate cooling. Agricultural and forest

[5]. Stanghellini C. 1987. Transpiration of greenhouse crops : An Aid to climate Management, ph D Thesis, Institut voor Mechanisatie, Agricultural University, Wageningen, Netherlands.

[6]. Bellache O. 1995. Serres Solaire, thermique appliquée aux zones arides.Première école d'été, Juillet, Agadir-Maroc.

[7]. Belhamri M. 1996. Terre et Vie, $\mathrm{N}^{0} 113$, Novembre.

[8]. Boulard T, Baille A. 1987. Analysis of thermal performance of greenhouse as a solar collector. Energy in Agricuture, vol. 6, pp. 1726

[9]. Enea G, Duplaix J, Balmat F, Bouchouicha M. 2001. Automatisation des cultures sous serres agricoles. Deuxième séminaire Tunisien d'automatique STA. 2001, pp.74-97, Douz, Tunisie. 